Proceedings of the 55th Hawaii International Conference on System Sciences | 2022

Minitrack: IT Adoption, Diffusion, and Evaluation in Healthcare

\author{
Akshat Kapoor \\ East Carolina University \\ kapoora16@ecu.edu
}

Arnold Kamis

Brandeis University

akamis@brandeis.edu

\section{Ton Spil}

University of Twente

a.a.m.spill@utwente.nl
Karoly Bozan

Duquesne University

bozank@duq.edu
The adoption, diffusion, and evaluation of IT in healthcare continue to present challenges to organizations and society, as well as to researchers. IT is seen as an enabler of change both globally and locally in healthcare organizations. However, IT adoption decisions in healthcare are complex because of the uncertainty of benefits and the rate of change of technology. This minitrack is one of the longest running minitracks at HICSS, this year celebrating our $20^{\text {th }}$ anniversary.

The papers in this minitrack utilize numerous research approaches. Delphi studies, surveys, interviews, and longitudinal case studies all provide different methods to investigate the range of issues underlying the successful adoption, implementation, and evaluation of IT. This minitrack contains a variety of interesting and discussion-provoking papers with some recurring themes.

Paper 3058 Predicting Medical Students' Intention to Integrate Digital Health into their Medical Practice: A Pre- and Post-COVID-19 Survey in Canada. The paper investigates how medical student characteristics predict the use of digital health (dHealth) and artificial intelligence into their future medical practices. The two-phased survey study of Canadian medical students empirically supported the proposed model that predicts the medical students' intention to integrate dHealth into their medical practices. The implications to incorporate dHealth in the medical education is discussed.

Paper 3358 Trust Recipes for Enhancing the Intention to Adopt Conversational Agents for Disease Diagnosis: An fsQCA approach. The paper investigates trust factors influencing the behavioral intent to adopt conversational agents for disease diagnosis. The authors use a medical diagnostic app that combines patients' symptoms with their medical history and provides diagnostic suggestions. They analyze their survey data using a fuzzy set qualitative comparative analysis, a configurational approach, and find that adoption intentions are strongly associated with performancerelated trust factors. Propositions are developed to help both researchers and practitioners.

Paper 3620 Influence of Personality Traits on the Continued Use of Fitness Apps. The paper examines post-adoption factors affecting the continued use or dropout among users of fitness apps using the expectation confirmation model, with a specific focus on the impact of users' personality traits. A survey of 129 participants revealed that conscientiousness and neuroticism as traits positively influence the perceived usefulness of fitness apps.

Paper 3849 How to turn the Fast-Track into a FastTrack: Process integration for evaluation of the quality of Digital Health Applications (DiGAs) on the example of the German Fast-Track Process. This paper addresses the research question of which integration points in the Itextit \{German Fast-Track process\} are particularly well suited for the integration of evaluation platforms for digital health applications. For this purpose, possible integration points are first identified and then analyzed with the help of a utility analysis with regard to the posed research question. Finally, a recommendation for action is made based on the results of the conducted utility analysis.

Paper 3850 Addressing unwarranted clinical variation in healthcare as a quality improvement process. With organizational support, the utilization of collaborative methodologies and the leveraging of available digital health technologies, healthcare organizations are provided the greatest opportunity for the reduction of unwarranted clinical variation and the optimization of healthcare outcomes. 
Paper 4085 An Institutional Theory Perspective on EHR Engagement: Mandates, Penalties, and Enforcement. The paper builds on the premise that user engagement with EHR systems differ from traditional IS context due to the highly regulated environment. The authors, cooperating with a leading healthcare analytics firm, identify unique roles in their model and propose a better model fit compared to those used in traditional technology acceptance studies.

Paper 4347 Investigating the impact of social support embedded in online consultation on physicians' online reputation. The paper investigates the relationship between physicians' computer-mediated social support and their online reputation. An analysis of online consultation records from a leading Chinese online consultation platform revealed that physicians' action-facilitating support and nurturant support had significant positive impacts on their online reputation.

Paper 4383 Where to Start with AI?Identifying and Prioritizing Use Cases for Health Insurance. The paper identifies and prioritizes AI use cases for health insurance in Germany based on technological, organizational, and environmental factors. The authors conduct interviews in the areas of predictive health, individualized service, anomaly detection, and operations enhancement. They find that the main dimensions are implementation complexity and expected value, with an emphasis on cost savings. The results help AI practitioners understand what is likely to be valued and prioritized.

Paper 4506 A Thematic Analysis of Provider Comparisons of Telemedicine Satisfaction Measures. The paper performs a thematic analysis of telemedicine satisfaction measures. The authors obtain qualitative feedback from telemedicine providers on their views of the results of different satisfaction, with a focus on non-numerical, humancentered data. The results included single- and multiple-dimension measures of satisfaction across eleven themes, which suggest the importance of allowing for subjective, experiential interpretations of success. Implications for researchers and providers are discussed.
The above 9 papers cover a wide range of challenges healthcare faces and they highlight possible solutions. We look forward to discussing these topics in this minitrack and encourage the authors to consider the feedback they receive advance their studies after the conference. Furthermore, we encourage submissions in the future addressing healthcare technology adoption, diffusion, and evaluation challenges using a variety of methods and research approaches. 